\title{
Estudio interuniversitario en estudiantes de Ciencias de la Salud: uso de los dispositivos móviles con fines educativos
}

Ángela González-Santos ${ }^{\mathrm{a}}$, Lucía Ortiz-Comino ${ }^{\mathrm{b}}$, Paula Postigo-Martín ${ }^{\mathrm{a}}$, María López-Garzón ${ }^{\mathrm{a}}$, Eva Serna $^{c}$, María Dolores Mauricio ${ }^{c}$, Teresa San-Miguel ${ }^{d}$, Mario Lozano-Lozano ${ }^{a}$

${ }^{a}$ Departamento de Fisioterapia, Facultad de Ciencias de la Salud, Universidad de Granada ${ }^{b}$ Departamento de Fisioterapia, Facultad de Ciencias de la Salud de Melilla, Universidad de Granada (luciaoc@ugr.es)

${ }^{c}$ Departamento de Fisiología, Facultad de Medicina y Odontología, Universitat de València ${ }^{\mathrm{d}}$ Departamento de Patología, Facultad de Medicina y Odontología, Universitat de València

\section{\$EWWFW}

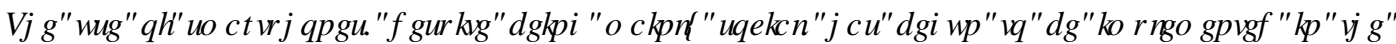
XQVH UИW HQVILRQP HQWDW D URROIRU Z RUNQJ IQ FQDW DQCI IRU FRP P XQIFDURQ EHZ HQ WXGHW

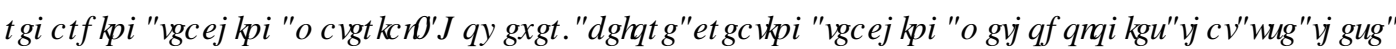

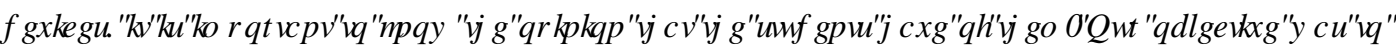

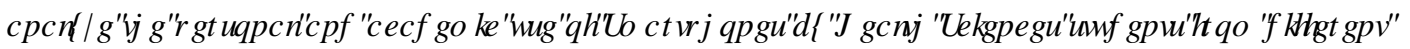

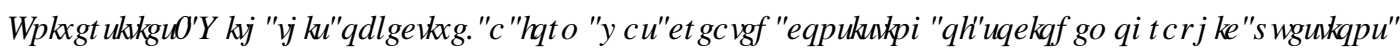

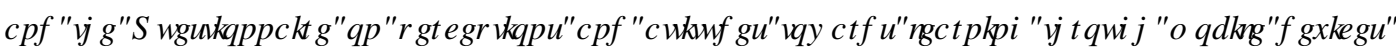

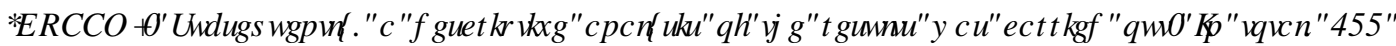

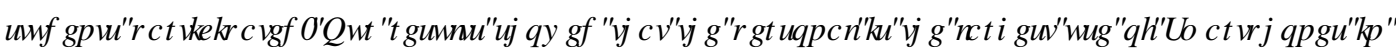

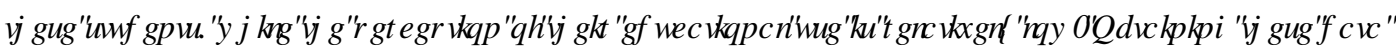

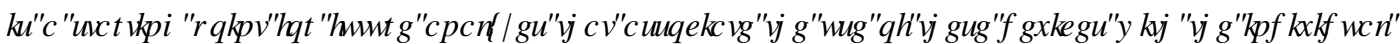

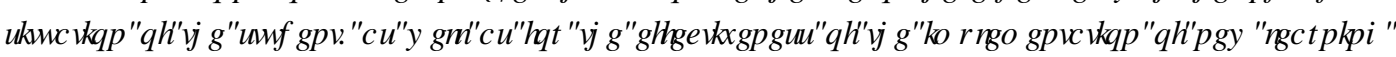
$P$ HKRGRQJIHM

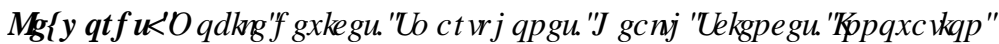

\section{HXP HQ}

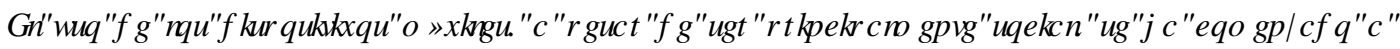

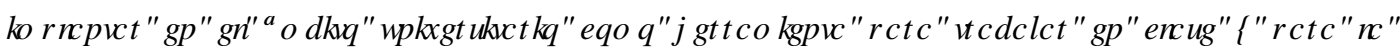

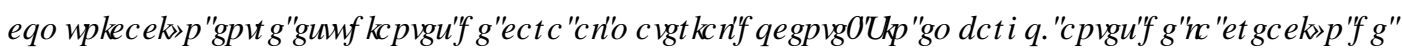

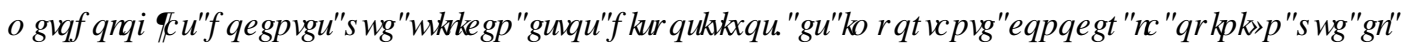

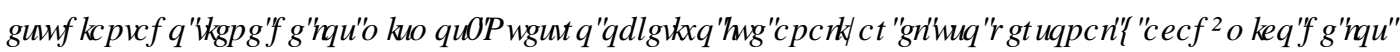

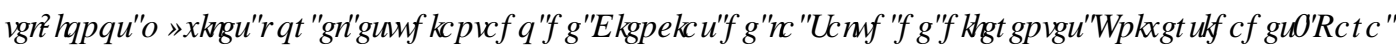

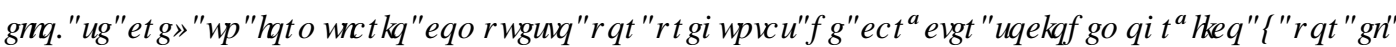

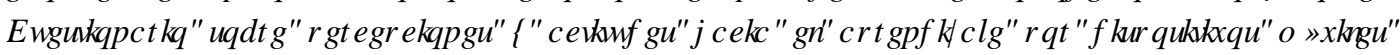

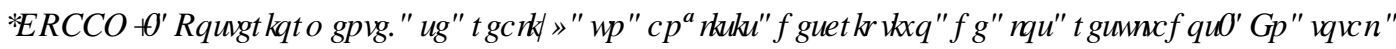

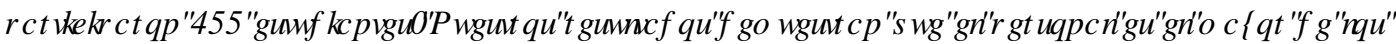

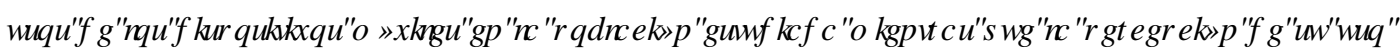

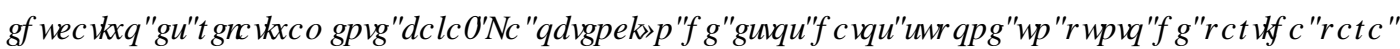

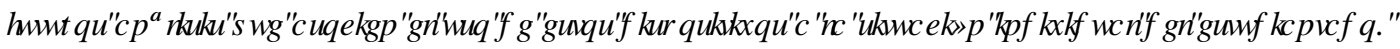

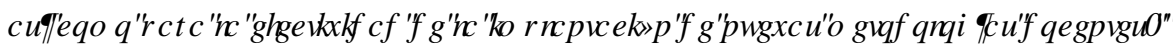

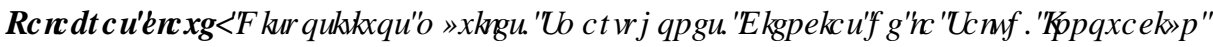




\section{Introducción}

En las últimas décadas, el uso de las nuevas tecnologías se ha ido expandiendo cada vez más en todas las

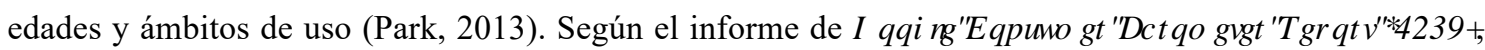
el $100 \%$ de las personas menores de 25 años señalan hacer un uso diario del teléfono móvil para navegar por Internet, al igual que el 98\% de las personas entre 25-34 años. Concretamente en nuestro país, el teléfono móvil o 6PDU\&KRQHes el dispositivo preferido por excelencia como vía de acceso a Internet entre la población joven y adulta, situándose por encima del ordenador y las tabletas electrónicas. Por lo tanto, se puede afirmar que el móvil forma parte de nuestro día a día en la actualidad por su capacidad de satisfacer las necesidades personales de seguridad, socialización, capacidad individual y aprendizaje.

En esta línea, la gran cantidad de información a la que estamos expuestos diariamente incrementa la dificultad para acceder a ella y procesarla adecuadamente. En el entorno educativo y, más concretamente el universitario, ocurre algo similar, por lo que es necesario dotar a los alumnos de habilidades para la selección, análisis y procesamiento de la información relevante. Esto conlleva la necesidad de un cambio en la metodología docente, en la que el alumno pasaría a tener un papel proactivo en el proceso de aprendizaje, sumado al hecho de asimilar los conocimientos transmitidos por el docente de forma tradicional (García-Valcárcel, 2007).

La necesidad de nuevos diseños educativos se relaciona con las nuevas tecnologías de última generación como vía eficaz para solventarlo (Viera-Santana, 2016). Estos dispositivos permiten aprovechar en el entorno educativo su potencial creativo, interactivo, comunicativo y documental. El uso de las nuevas tecnologías en el aula ha evolucionado desde el uso de ordenadores hasta el uso del teléfono móvil, considerado como " $P \square$ ADLQJ" " ppor su nombre en inglés. Esta metodología permite aprender a través de dispositivos que una persona puede portar en el bolsillo, pudiendo así utilizarlo en cualquier momento y cualquier lugar. Además, permite el acceso a la educación en casos en los que factores de tipo cultural, económico, social o geográfico suponen una dificultad para ello (Traxler, 2010).

Algunos autores destacan, sin embargo, las consecuencias negativas a nivel académico que puede suponer el uso de los dispositivos móviles para los estudiantes, como la pérdida de concentración y la procrastinación (Rozgonjuk, 2018). No obstante, otros autores afirman que el hecho de incluir actividades educativas en el aula a través de la tecnología móvil ayuda a incrementar la motivación y participación de los estudiantes, así como sus resultados académicos (Kilis, 2013; Fernández-Lao, 2016). Es aquí donde la labor del docente juega un papel importante como mediador del aprendizaje para garantizar que esta práctica se dirija hacia la mejora de la calidad de la educación, comportamiento y calidad de vida de los estudiantes. Además, los estudiantes universitarios suelen utilizar frecuentemente el teléfono móvil en el aula y fuera de ella con fines no solo sociales sino también educativos, principalmente en actividades tales como el intercambio de información académica, coordinación de trabajos grupales y consulta de servicios universitarios (Vázquez-Cano, 2015). De hecho, se ha demostrado que el uso del móvil aporta beneficios en la mejora de la comunicación y retroalimentación de los estudiantes que lo usan frente a los que no (Silva, 2017).

Varios autores han coincidido en la necesidad de diseñar aplicaciones móviles para utilizar en el entorno universitario (Kilis, 2013), concretamente en la formación de profesionales de la salud (Dunleavy, 2019). Asimismo, la Unesco ha considerado el teléfono móvil como una de las herramientas educativas con mayor proyección en los países en vías de desarrollo (Unesco, 2013). Sin embargo, no todos los centros educativos ni todos los docentes han dado este salto de calidad, manteniéndose en el uso de programas de ofimática tradicionales. Por lo tanto, es imprescindible la innovación en este campo para incrementar su evidencia, así como la visión de los propios alumnos sobre el uso de estos dispositivos en el entorno universitario con fines académicos.

(c)) EY-NC-ND 2021, Universitat Politècnica de València

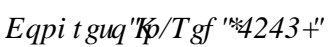




\section{Objetivos}

El objetivo general de este estudio es analizar de manera global el uso general y académico del teléfono móvil entre los estudiantes de diferentes grados de Ciencias de la Salud de tres universidades españolas: la Universidad de Almería, la Universidad de Granada y la Universitat de València.

\section{Desarrollo de la innovación.}

Durante el mes de octubre de 2020, profesorado perteneciente a diferentes universidades nos pusimos en contacto para comenzar a trabajar sobre el desarrollo de un proyecto de innovación a largo plazo en el que poder abarcar diferentes dimensiones del estudiantado de Ciencias de la Salud. Finalmente, se decidió abordar, en una primera fase, el uso de los dispositivos móviles tanto en su vida personal como a nivel universitario, con la intención de, en un futuro próximo, crear herramientas docentes que tengan en cuenta la predisposición del alumnado al uso de nuevas tecnologías en la educación. Para recoger esta información, se creó un formulario a través de la herramienta homónima de Google, la que permite un acceso libre y un fácil manejo de los datos para su posterior análisis.

Una vez creada la herramienta de trabajo, el siguiente paso fue recoger datos de las diferentes Universidades en las que el profesorado que conforma este proyecto imparte docencia: Universidades de Almería (UAL), Granada (incluido su campus ubicado en Melilla) (UGR) y Universitat de València (UV). Este proceso comenzó durante el inicio del segundo semestre del curso 2021. Para ello, durante el inicio de la clase, se compartió con el alumnado el enlace al formulario a través de la plataforma virtual de videoconferencia mediante la que se imparten actualmente las clases de docencia teórica.

Antes de completar el formulario, se indicaron a los participantes los objetivos de su uso, las partes de las que estaba compuesto y el tiempo aproximado para completarlo. Además, se les informó de que completar el formulario era completamente voluntario. Para asegurar su consentimiento de cara a su participación, se incluyó una primera pregunta, solicitándoles su consentimiento para participar en el mismo. Igualmente, si durante la realización del mismo les surgían dudas, podían comunicárnoslas a través de la herramienta de videoconferencia. También se les ofreció la posibilidad de enviarles los resultados individuales una vez analizados.

Específicamente, el formulario se compone de dos apartados claramente definidos: por un lado, recogimos datos sociodemográficos generales (edad, sexo, universidad a la que pertenecen) y que pudieran suponer un mayor o menor uso de los dispositivos móviles (grado y año que cursan actualmente, número de matrícula de la asignatura donde se compartió el formulario, compaginación con actividades laborales a tiempo completo o parcial y dependencia económica de becas para el estudio); por otro lado, se utilizó el cuestionario sobre percepciones y actitudes hacia el aprendizaje por dispositivos móviles (CPAAM). Este cuestionario fue desarrollado por Seifert y colaboradores (Seifert, 2019), con el objetivo de crear un documento válido y fiable a la hora de evaluar el aprendizaje a través de los dispositivos móviles, partiendo de la percepción y la actitud que presenta el alumnado de cara a estos dispositivos. Consta de 40 ítems divididos en 4 dimensiones, a saber: (1) usos lucrativos y/o personales, (2) usos educativos, (3) percepciones y actitudes hacia los dispositivos móviles y (4) ventajas y riesgos de los dispositivos móviles. Cada ítem se evalúa a través de una escala Likert de 5 puntos, desde 1 (completamente en desacuerdo) hasta 5 (completamente de acuerdo). Para calcular el valor de cada una de las dimensiones, se calcula la media aritmética de la misma. 
Finalmente, recogidos todos los datos, se volcaron a una base de datos a través del programa SPSS v 24.0 para el posterior análisis de los mismos. Se realizó un análisis descriptivo, expresando los datos continuos en valores de medias y desviaciones estándar y en porcentajes los datos de carácter categórico.

\section{Resultados}

En total, 233 estudiantes participaron en este estudio preliminar, de los cuales el $57.5 \%(\mathrm{n}=134)$ pertenecían a la Universitat de València, y de este porcentaje, un $6.7 \%(n=9)$ estaban matriculados en un grado mixto compartido con la Universitat Politècnica de València; el $24.9 \%(n=58)$ a la Universidad de Granada, el $16.7 \%(\mathrm{n}=39)$ a la Universidad de Almería y el resto, un $0.9 \%(\mathrm{n}=2)$ a la Universidad de Verona (Italia), al encontrarse durante el curso actual realizando una estancia Erasmus + en la Universidad de Almería. Con una edad media de $21.39 \pm 5.88$, el $68.2 \%$ eran mujeres $(n=159)$ y el $30.9 \%(n=72)$ hombres. Solo un $0.9 \%$ no se identificaba con alguna de las etiquetas de género socialmente establecidas. El estudiantado que participó pertenecía a los grados de Fisioterapia (45.1\%, n=105), Medicina (33.9 \%, $\mathrm{n}=79)$, Enfermería (16.3\%, $\mathrm{n}=38)$, Ingeniería Biomédica $(3.9 \%, \mathrm{n}=9)$ y Trabajo Social $(0.9 \%, \mathrm{n}=2)$. Solo un $36.1 \%(n=84)$ del estudiantado estaba cursando las asignaturas que se integran en este estudio como primera matrícula, por lo que el $63.9 \%(\mathrm{n}=149)$ se encontraban en su segunda matrícula o posteriores. Con respecto a la situación económica, el $76.4 \%(\mathrm{n}=178)$ de los participantes se encontraban en situación de desempleo y sin búsqueda activa de empleo, mientras que un $9.9 \%(n=23)$ refirieron encontrarse también en situación de desempleo pero en búsqueda del mismo. A pesar de que un $22.3 \%$ $(\mathrm{n}=52)$ del estudiantado refirió depender económicamente de una beca, el $51.1 \%(\mathrm{n}=119)$ recibió dicha beca, del cual, al $55 \%$ solo le cubría la matrícula universitaria, mientras que al $45 \%$ también incluía algún tipo de ayuda para la manutención.

Con respecto al uso de dispositivos móviles tanto dentro como fuera del aula, en la figura 1 se pueden observar los datos recogidos mediante el cuestionario sobre percepciones y actitudes hacia el aprendizaje por dispositivos móviles (CPAAM). En cuanto al uso lucrativo y/o personal de estos dispositivos, parece que la actual generación de estudiantes los utiliza por igual independientemente de la opción estudiada, a excepción de su uso como elemento de recreación mediante videojuegos móviles, que recibe la puntuación más baja $(2.88 \pm 1.51)$. Para el subapartado de usos educativos, parece que el estudiantado valora positivamente el uso de los móviles como herramienta de comunicación con el profesorado (4.12 \pm 1.19) y para la descarga de documentación relacionada con el curso ( $3.99 \pm 1.29)$; mientras que el empleo del dispositivo móvil para hacer uso de los servicios de la biblioteca $(2.60 \pm 1.51)$ o la grabación de voz en el aula $(2.18 \pm 1.47)$ son los peor valorados. En cuanto a la percepción y actitud hacia el dispositivo móvil, la puntuación más elevada fue para la pregunta de realizar tareas de forma más rápida (4.46 \pm $0.83)$, mientras que la peor puntuación fue otorgada a la opción de discusión en foros (2.42 \pm 1.30$)$. Finalmente, en cuanto a la percepción de ventajas y riesgos de los usos, cabe destacar la opción más puntuada, crear adicción $(4.8 \pm 0.48)$ fomento de la desconcentración $(4.47 \pm 0.83)$ o incentivar al aislamiento $(4.31 \pm 0.93)$ mientras que la menos puntuada es la de mejorar las relaciones sociales $(2.99 \pm$ 1.37). 


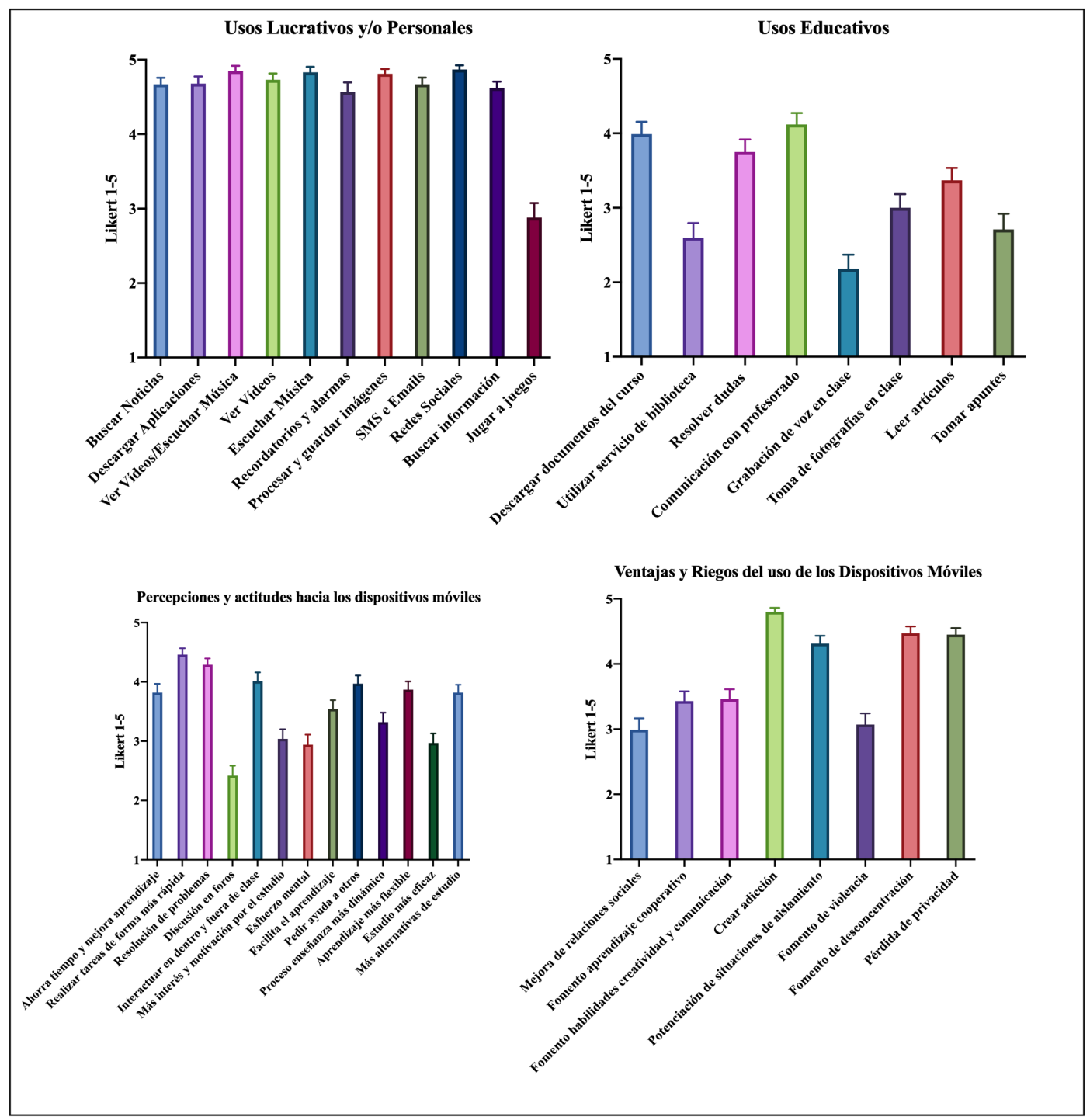

Figura 1: Cuestionario sobre Percepciones y Actitudes hacia el Aprendizaje por dispositivos Móviles (CPAAM)

En la figura 2, se observa la puntuación global en cada subescala del CPAAM. En ella se puede observar como el uso lucrativo y personal es la mayor utilidad de los dispositivos móviles percibida entre el alumnado de Ciencias de la Salud. Sin embargo, aun en la actualidad, y en un contexto de pandemia con muchas clases virtualizadas, la percepción del uso de estos dispositivos con fines educativos sigue recibiendo una puntuación relativamente baja.

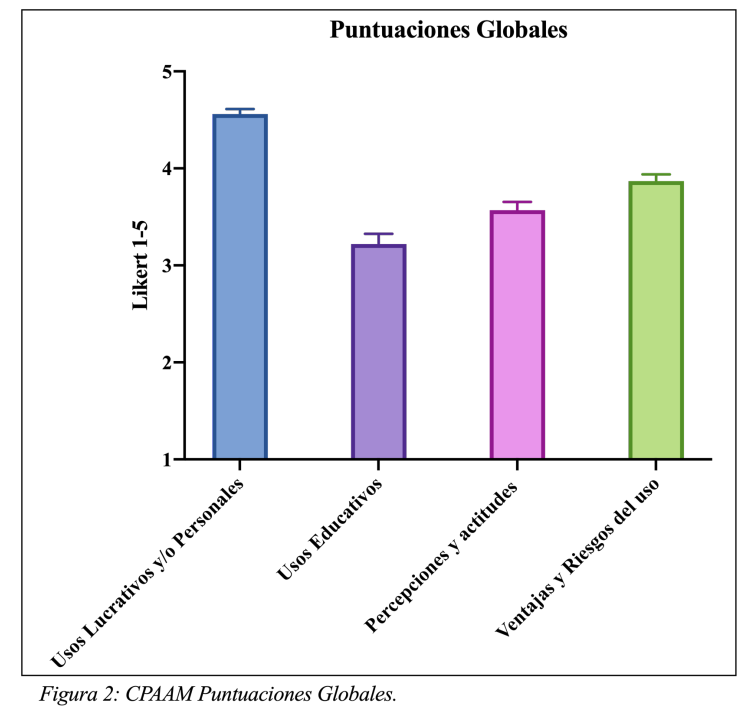




\section{Conclusiones}

En base a los resultados obtenidos, parece sensato pensar que el uso de los dispositivos móviles en el aula puede ser una herramienta útil y necesaria, a la vez que bien aceptada por parte del alumnado. Sin embargo, sorprende la percepción de los riesgos del uso de estos dispositivos entre el estudiantado, siendo conscientes de su aspecto negativo en cuanto a las relaciones sociales o el fomento del aislamiento social o incluso de la pérdida de la privacidad. Sin embargo, a pesar de percibir los riesgos de las mismas, los usos más extendidos siguen siendo los lucrativos, entre los que destaca el uso de las redes sociales. Además, en cuanto a su uso en clase cabe destacar que, a pesar de haber recibido una baja puntuación, el uso del móvil para grabar en el aula recibe una puntuación excesivamente elevada si tenemos en cuenta la actual política de las universidades implicadas, que prohíben su uso con dichos fines en clase.

La obtención de estos datos supone un punto de partida para futuros análisis que asocien el mayor o menor uso de los dispositivos móviles en función de la situación individual del estudiantado, así como la efectividad de la implantación de nuevas metodologías docentes en las que se utilicen los dispositivos móviles como herramienta principal.

\section{Referencias}

DUNLEAVY, G., NIKOLAOU, CK., NIFAKOS, S., ATUN R., CHUN YI LAW, G., TUDOR CAR, L. (2019). "Mobile digital education for health professions: systematic review and meta-analysis by the digital health education collaboration" en Journal of Medical Internet Research, vol. 12, issue 21.

FERNÁNDEZ-LAO, C., CANTARERO-VILLANUEVA， I., GALIANO-CASTILLO, N., CAROMORÁN, E., DÍAZ-RODRÍGUEZ, L., ARROYO-MORALES, M. (2016). "The effectiveness of a mobile application for the development of palpation and ultrasound imaging skills to supplement the traditional learning of physiotherapy students" en $1 \%$ \& $\$$ HOFDO GXFDMRQ vol. 16, issue 1., p. 1-7.

GARCÍA-VALCÁRCEL, A. (2007). "Herramientas tecnológicas para mejorar la docencia universitaria. Una reflexión desde la experiencia y la investigación" en 5HLWD, EHRDP HUFDQD GH ( GXFDFIyQD ' IMLOFID, vol. 10, issue 2, p. 125-148.

KILIS, S. (2013). "Impacts of mobile learning in motivation, engagement and achievement of learners: review of literature" en * D]IDQUAБ

PARK, N., KIM, Y.C., SHON, H.Y., SHIM, H. (2013). "Factors influencing smartphone use and dependency in South Korea” en \&RP SXWHWIQ+ XP DQ\%HDVIRUlvol. 29, issue 4, p. 1763-1770.

ROZGONJUK, D., KATTAGO, M., TÄHT, K. (2018). "Social media use in lectures mediates the relationship between procrastination and problematic smartphone use" en \&RP SXWAWIQ + XP DQ\% HOVIRU vol. 89, p. 191-198.

SEIFERT, T., HERVÁS GÓMEZ, C., TOLEDO MORALES, P (2019). "Diseño y validación del cuestionario sobre percepciones y actitudes hacia el aprendizaje por dispositivos móviles" en $\square 3$ L[HD\%dW 5 HILDQGHO HGIRVI \&( GXFDFlyQ vol. 54, p. 45-64. 
SILVA CALPA, AC., MARTÍNEZ DELGADO, DG. (2017). "Influencia del smartphone en los procesos de aprendizaje y enseñanza" en 6XP DGHQH RFIRV, vol. 8, p. 11-18

TRAXLER, J. (2010). "Distance education and mobile learning: Catching up, taking stock" en ' LMDQRH ( GXFDMRQ vol. 31, issue 2, p. 129-138.

UNESCO (2013). "Unesco Policy Guidelines for Mobile Learning”. En: París: United Nations Educational, Scientific and Cultural Organization

VÁZQUEZ-CANO, E., SEVILLANO-GARCÍA, ML. (2015). "El smartphone en la educación superior. Un estudio comparativo del uso educativo, social y ubicuo en universidades españolas e hispanoamericanas" en 6LJQR』 [3 HQMPP IHQWR, vol. 34, issue 67, p. 114-131.

VIERA-SANTANA, JG., HERNÁNDEZ-HADDAD, J.C., CASTILlO-ORTIZ, J., DOMÍNGUEZRODRÍGUEZ, R. (2016) "Las tecnologías móviles en ámbito universitario". En: ,,, , -RLQDODV ,EHRDP HUFDQDVGH, QQRYDFIYQ( GXFDUMDHQHDi P EIMRIGHIDV7, \&. Las Palmas de Gran Canaria. 3-10. 